\title{
A Novel Approach to Teaching Empathy to Pharmacy Students
}

Authors: Angela Macadam and S.Williams, School of Pharmacy and Biomolecular Sciences, University of Brighton, UK

Background: Empathy is an important factor in patient care however, studies suggest that pharmacists have lower empathy scores than GPs and pharmacy students have lower scores than medical students ${ }^{1}$. It has been suggested that teaching empathy is difficult and is not being taught consistently to pharmacy undergraduates ${ }^{2}$. The aim of this project was to teach empathy through workshops and evaluate their value.

Method: Two workshops were developed in consultation with trained actors to teach students how to get into another person's role. All pharmacy students at this University were invited, by e-mail, to take part in the workshops.

The workshops were attended by the students, two academic tutors and two actors who led the workshops. Following the workshops, everyone involved was interviewed about the project.

Results: Out of 367 students, eight students attended the workshops.

Tutors' reflections: "This was a completely different way of approaching communications skills training." Generally I believe that this type of patient modelling is a useful teaching tool."

Students' reflections: "I don't think many students turned (up) because the workshops ran during the inter-semester break when many students had exams." "The acting of the 'worst patient in the world' was a brilliant way of seeing how to deal with difficult patients."

Actors' reflections: "The Role-play and character development worked well everyone engaged." "The task of coming up with and playing a role on the spur of the moment was quite challenging for some students."

Conclusions: This was an interesting novel approach to teach empathy to pharmacy students. The main limitation was the small number of participants. This could be addressed in the future by fitting the workshops into an existing module. Further work would be needed to establish if this form of teaching does enhance empathy among students.

\section{References}

1. Varsani S, Ellis-Martin M, MacAdam AB. How empathic are pharmacists? Int J Pharm Prac. 2006 14(1): A30

2. Van Winkle LJ, Fjortoft $\mathrm{N}$ and Hojat $\mathrm{M}$. Impact of a workshop about aging on the empathy scores of pharmacy and medical students. Am J Pharm Educ 2012 76(1) 9 